\title{
Observations of three pre-cataclysmic variables from the Edinburgh-Cape blue object survey
}

\author{
C. Tappert ${ }^{1, \star}$, B. T. Gänsicke ${ }^{2}$, M. Zorotovic ${ }^{1}$, I. Toledo ${ }^{1}$, J. Southworth ${ }^{2}$, C. Papadaki ${ }^{3}$, and R. E. Mennickent ${ }^{4}$ \\ 1 Departamento de Astronomía y Astrofísica, Pontificia Universidad Católica, Vicuña Mackenna 4860, $782-0436$ Macul, Chile \\ e-mail: ctappert@dfa.uv.cl,mzorotov@astro.puc.cl,itoledoc@gmail.com \\ 2 Department of Physics, University of Warwick, Coventry CV4 7AL, UK \\ e-mail: [Boris.Gaensicke; j.k.taylor]@warwick.ac.uk \\ 3 Vrije Universiteit Brussel, Pleinlaan 2, 1050 Brussels, Belgium \\ e-mail: cpapadak@vub.ac.be \\ 4 Departamento de Astronomía, Universidad de Concepción, Casilla 160-C, Concepción, Chile \\ e-mail: rmennick@astro-udec.cl
}

Received 12 March 2009 / Accepted 20 May 2009

\section{ABSTRACT}

\begin{abstract}
Aims. This study aims at determining the parameters of the three candidate pre-cataclysmic binaries EC 12477-1738, EC 133493237, and EC 14329-1625, most importantly their orbital period.

Methods. Time-series photometry reveals orbital modulation in the form of sinusoidal variation due to the reflection effect. Photometric observations are complemented by time-resolved spectroscopy that yields radial velocities of the $\mathrm{H} \alpha$ emission line. The combination of both methods allows us to unambiguously determine the orbital periods. The average spectra are used to estimate physical parameters of the primary and secondary stellar components.

Results. We determine the orbital period for EC $12477-1738$ as $0.362 \mathrm{~d}$, thus confirming the value previously reported. A similar period, $P=0.350 \mathrm{~d}$, is found for EC 14329-1625. Both systems incorporate a medium-hot white dwarf $(T=15000-20000 \mathrm{~K})$ and an M3V secondary star. The third pre-CV, EC 13349-3237, is the youngest of the three, with a hot WD ( $T \sim 35000 \mathrm{~K})$, and it also has the longest period $P=0.469 \mathrm{~d}$. It furthermore turns out to be one of the still rare pre-CVs with a comparatively early-type, M1V, secondary star, which will eventually evolve into a CV above the period gap.
\end{abstract}

Key words. binaries: close - stars: late-type - white dwarfs - novae, cataclysmic variables

\section{Introduction}

Cataclysmic Variables (CVs) are close interacting binaries with a white dwarf accreting material from a Roche-lobe filling, latetype (K-M), main-sequence star. These systems are thought to form from initially separated binaries that go through a commonenvelope phase as the more massive one of the two stars expands in the course of its nuclear evolution. The eventual expulsion of the common-envelope material leaves a still separated white dwarf / main-sequence star (hereafter WD/MS) binary. Angular momentum loss due to magnetic braking and gravitational radiation shrinks the separation, which eventually leads to the secondary star filling its Roche lobe, thus starting mass-transfer. Following Schreiber \& Gänsicke (2003), we call the WD/MS binaries pre-CVs, if the evolution from the expulsion of the common envelope to the start of mass transfer takes place within Hubble time ( 13 Gyr).

Recent attempts to solve the discrepancies between the modelled and the observed CV population (e.g., Stehle et al. 1997; Patterson 1998; Gänsicke 2005) emphasise the importance of the pre-CV phase (Schenker \& King 2002; Schenker et al. 2002). The detection of anomalous element abundances in a number of CVs (Gänsicke et al. 2003; Harrison et al. 2004, 2005) raises the question of whether the secondary star undergoes a certain

* New address: Departamento de Física y Astronomía, Universidad de Valparaíso, Avenida Gran Bretaña 1111, Valparaíso, Chile amount of nuclear evolution prior to entering the CV stage, although an examination of 13 pre-CVs gave a negative result (Tappert et al. 2007b).

Since pre-CVs consist of two intrinsically faint stellar components and - with the exception of eclipsing systems and binary central stars of planetary nebulae - show only very minor photometric variability (with amplitudes of typically $\leq 0.1 \mathrm{mag}$ ), they are not easily detected. Consequently the number of known preCVs is small; applying the criteria from Schreiber \& Gänsicke (2003), i.e., $P_{\text {orb }}<2$ d and a main-sequence secondary with $M_{2}<M_{1}$, we find 56 potential and confirmed pre-CVs in version 7.9 of the Ritter \& Kolb (2003) catalogue. The Sloan Digital Sky Survey (SDSS) is currently fundamentally changing this picture, thanks to the vast number of spectroscopic follow-ups of point sources with non-stellar ugriz colours (Raymond et al. 2003; Silvestri et al. 2006, 2007; Rebassa-Mansergas et al. 2007; Schreiber et al. 2008), and detailed follow-up observations will eventually provide $\simeq 2000$ new systems. However, the majority of the SDSS systems will be faint, requiring large-aperture telescopes for follow-up observations.

Here, we present photometric and spectroscopic studies of three relatively bright pre-CVs, EC 12477-1738, EC 133493237, and EC 14329-1625, which were discovered and classified as WD/MS binaries in the Edinburgh-Cape Blue Object Survey (Kilkenny et al. 1997). Coordinates and apparent magnitudes of these systems are collected in Table 1; finding charts 


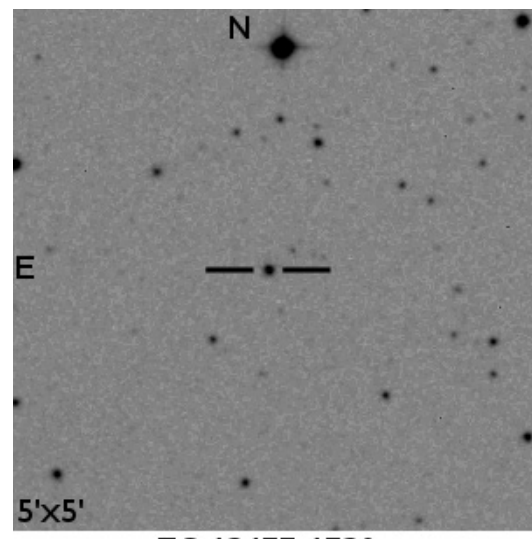

EC 12477-1738

$R A=12: 50: 22.1, D E C=-17: 54: 47$ (J2000.0)

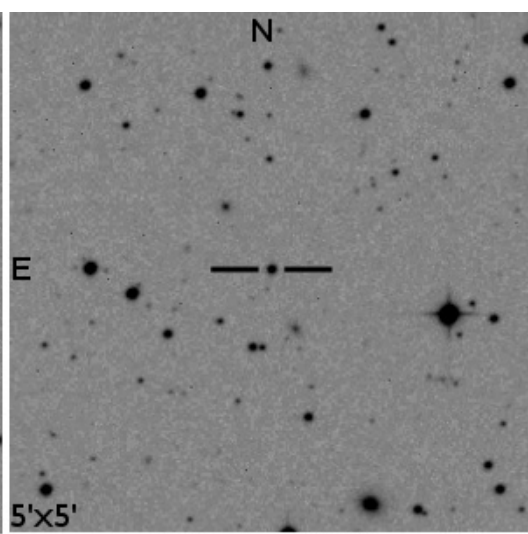

EC 13349-3237 (J2000.0)

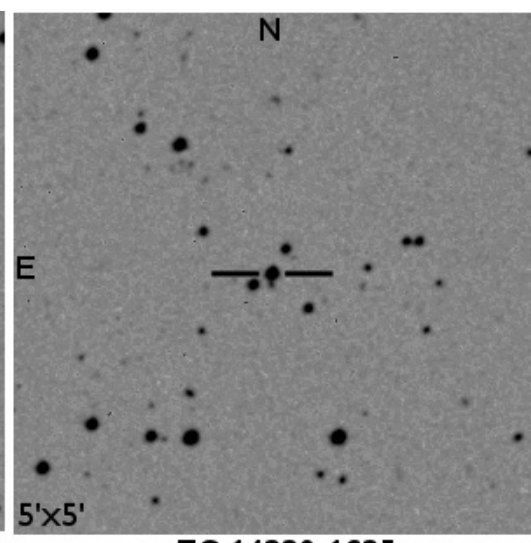

EC 14329-1625

(J2000.0)

Fig. 1. Finding charts from the Digital Sky Survey (DSS2-red).

Table 1. General information on the three targets of this study.

\begin{tabular}{llll}
\hline \hline Object & RA $(2000.0)^{1}$ & Dec $(2000.0)^{1}$ & $V[\mathrm{mag}]^{2}$ \\
\hline EC 12477-1738 & $12: 50: 22.1$ & $-17: 54: 47$ & 16.2 \\
EC 13349-3237 & $13: 37: 50.8$ & $-32: 52: 23$ & 16.3 \\
EC 14329-1625 & $14: 35: 45.7$ & $-16: 38: 17$ & 14.9 \\
\hline
\end{tabular}

1) from the SIMBAD database; 2) from Kilkenny et al. (1997)

are presented in Fig. 1. The first attempts to derive their orbital period photometrically have been described in Tappert et al. (2004) and Tappert et al. (2006). Maxted et al. (2007) used timeresolved spectroscopy to determine the period for one of these systems, EC 12477-1738.

\section{Observations and data reduction}

The photometric data were taken on several occasions in 2003 and 2005 at the $0.9 \mathrm{~m}$ CTIO/SMARTS telescope using an $R$ filter. The 2003 observations were part of a survey on a sample of 16 objects that had been classified as candidate pre-CVs based on their spectral appearance. The aim was to examine them for potential photometric variability that would confirm their classification and reveal their orbital period (Tappert et al. 2004). Since it was unknown if the targets would show any variation, continuous light curves were taken, i.e. a certain fraction of the night (usually around $4 \mathrm{~h}$ ) would be dedicated to a specific object. For the 2005 observations, the sample was limited to 5 objects with known or suspected light curve modulations corresponding to periods $>6 \mathrm{~h}$. Thus, targets were cycled throughout the night, with three consecutive data points being taken per step. In this way, light curves covering $\sim 8 \mathrm{~h}$ per night could be measured for three to four targets, yielding a time resolution of $\sim 0.5 \mathrm{~h}$. Unfortunately, the 2005 observing runs were plagued with bad weather conditions, so that in the end only the data for the brightest target, EC 14329-1625, proved useful.

From the photometric observations, four systems emerged as confirmed pre-CVs: LTT 560 (Tappert et al. 2007a) and the three targets of the present paper. The latter were selected for timeresolved spectroscopy on 3 nights in April 2007 at the $4.0 \mathrm{~m}$ CTIO in order to pin down the orbital period, since the photometry on its own does not allow for an unambiguous distinction between sinusoidal (one maximum per orbit due to the secondary star being irradiated by a hot white dwarf) or ellipsoidal variation (two maxima per orbit due to the deformed secondary star). We employed the R-C spectrograph and grating KPGL3 with a $1.0^{\prime \prime}$ slit to yield a wavelength range of $3565-7240 \AA$ at a spectral resolution of $4.8 \AA$. Using essentially the same strategy as for the 2005 photometric observations, after each target spectrum, a HeAr lamp wavelength calibration exposure was taken, and afterwards the telescope was pointed to the next object. Flux calibration standards LTT 4816 and LTT 7379 were observed at the beginning of night 1 and at the end of night 3, respectively. Since the weather conditions were not photometric (in fact, the middle night of our observing run was completely overcast), one expects the respective calibrations not to be very accurate. However, as we will see below, they still provide valuable information. A summary of the observations is given in Table 2.

Basic reduction of the photometric and spectroscopic data followed standard procedures for bias subtraction and flat fielding, using IRAF ${ }^{1}$ tasks. The majority of the photometric data was analysed with IRAF's apphot/daophot packages, and the standalone daomatch and daomaster routines (Stetson 1992). The final aperture radius for the photometry was chosen as the one that gave a minimum noise in the differential light curve of two non-variable field stars with magnitudes similar to the target. All field star photometry of a single CCD frame were then combined to give an average light curve, with variable and noisy stars being iteratively excluded. The differential light curve for the target finally was computed by subtracting the averaged field stars from the target data. In an attempt to reduce the noise in the light curves, an iterative weighting algorithm (Broeg et al. 2005) was used to compute the average comparison light curve for the April 2005 data. However, the gain in signal-to-noise ratio $(\mathrm{S} / \mathrm{N})$ proved insufficient to justify a re-evaluation of the other data sets.

The spectroscopic data were optimally extracted (Horne 1986) and wavelength and flux calibrated. Radial velocities of the $\mathrm{H} \alpha$ emission line (and of absorption lines of the secondary star, when possible) were measured by fitting single Gaussians to the line profile. The WD absorption lines were found to be too broad and the $\mathrm{S} / \mathrm{N}$ too low in order to be measured either by fitting or by using cross-correlation methods. Additionally, narrow emission lines were present in the centre of the Balmer absorption in all cases. The $\mathrm{H} \alpha$ radial velocities were corrected for the

${ }_{1}$ NOAO PC-IRAF Revision 2.12.2-EXPORT. 
Table 2. Log of observations.

\begin{tabular}{|c|c|c|c|c|c|c|c|}
\hline Object & Date $^{1}$ & $\mathrm{HJD}^{1}$ & Telescope & Configuration $^{2}$ & $n_{\text {data }}$ & $t_{\exp }[\mathrm{s}]$ & $\Delta t[\mathrm{~h}]$ \\
\hline \multirow[t]{5}{*}{ EC $12477-1738$} & 2003-04-08 & 2452738 & $0.9 \mathrm{~m}$ & $\mathrm{CCD}+R$ & 120 & $60 / 90$ & 3.35 \\
\hline & 2003-04-09 & 2452739 & $0.9 \mathrm{~m}$ & $\mathrm{CCD}+R$ & 70 & $90 / 180$ & 4.08 \\
\hline & 2003-04-10 & 2452740 & $0.9 \mathrm{~m}$ & $\mathrm{CCD}+R$ & 60 & 180 & 3.84 \\
\hline & 2007-04-03 & 2454194 & $4.0 \mathrm{~m}$ & KPGL3, 3565-7240, 4.8 & 7 & $900 / 1200$ & 8.28 \\
\hline & 2007-04-05 & 2454196 & $4.0 \mathrm{~m}$ & KPGL3, 3565-7240, 4.8 & 9 & $900 / 1200$ & 8.97 \\
\hline \multirow[t]{6}{*}{ EC 13349-3237 } & $2003-05-15$ & 2452775 & $0.9 \mathrm{~m}$ & $\mathrm{CCD}+R$ & 50 & $180 / 240$ & 4.57 \\
\hline & 2003-05-16 & 2452776 & $0.9 \mathrm{~m}$ & $\mathrm{CCD}+R$ & 47 & 240 & 3.68 \\
\hline & $2003-06-20$ & 2452811 & $0.9 \mathrm{~m}$ & $\mathrm{CCD}+R$ & 81 & 240 & 6.28 \\
\hline & 2003-06-21 & 2452812 & $0.9 \mathrm{~m}$ & $\mathrm{CCD}+R$ & 77 & 240 & 5.98 \\
\hline & 2007-04-03 & 2454194 & $4.0 \mathrm{~m}$ & KPGL3, 3565-7240, 4.8 & 7 & $900 / 1200$ & 7.59 \\
\hline & 2007-04-05 & 2454196 & $4.0 \mathrm{~m}$ & KPGL3, 3565-7240, 4.8 & 9 & $900 / 1200$ & 8.67 \\
\hline \multirow[t]{9}{*}{ EC 14329-1625 } & 2003-06-18 & 2452809 & $0.9 \mathrm{~m}$ & $\mathrm{CCD}+R$ & 119 & 90 & 4.16 \\
\hline & 2003-06-19 & 2452810 & $0.9 \mathrm{~m}$ & $\mathrm{CCD}+R$ & 120 & 90 & 4.54 \\
\hline & 2005-04-25 & 2453486 & $0.9 \mathrm{~m}$ & $\mathrm{CCD}+R$ & 9 & 150 & 3.57 \\
\hline & 2005-04-28 & 2453489 & $0.9 \mathrm{~m}$ & $\mathrm{CCD}+R$ & 27 & 150 & 8.35 \\
\hline & 2005-04-29 & 2453490 & $0.9 \mathrm{~m}$ & $\mathrm{CCD}+R$ & 36 & 150 & 7.88 \\
\hline & $2005-05-24$ & 2453515 & $0.9 \mathrm{~m}$ & $\mathrm{CCD}+R$ & 12 & $150 / 300$ & 5.14 \\
\hline & $2005-05-26$ & 2453517 & $0.9 \mathrm{~m}$ & $\mathrm{CCD}+R$ & 14 & 180 & 4.95 \\
\hline & 2007-04-03 & 2454194 & $4.0 \mathrm{~m}$ & KPGL3, 3565-7240, 4.8 & 7 & $600 / 900$ & 7.64 \\
\hline & 2007-04-05 & 2454196 & $4.0 \mathrm{~m}$ & KPGL3, 3565-7240, 4.8 & 8 & $600 / 900$ & 8.37 \\
\hline
\end{tabular}

1) start of night; 2) grism/grating, wavelength range, and spectral FWHM resolution in $\AA$.

motion with respect to the local standard of rest (LSR) as defined in IRAF's rvcorrect task (the Sun's velocity vector relative to the LSR being $\left.v_{\odot}=20 \mathrm{~km} \mathrm{~s}^{-1}, \alpha_{1900}=18 \mathrm{~h}, \delta_{1900}=30^{\circ}\right)$ and a potential instrumental flexure was accounted for by subtracting the variation of the $[\mathrm{O}$ I] $\lambda 5577$ night sky emission line.

Both the photometric and the spectroscopic data were examined for periodic modulation applying several routines implemented in the ESO-MIDAS ${ }^{2}$ time-series analysis (tsa) context, namely the Scargle (1982) and analysis-of-variance (AOV; Schwarzenberg-Czerny 1989) algorithms, as well as the extension of the latter, which uses orthogonal trigonometric polynomials to fit the phase-folded data (ORT; Schwarzenberg-Czerny 1996). After discarding alias periods (see the respective sections for each system), the error associated with the selected period was calculated using Eq. (4) from Larsson (1996).

\section{Results}

\subsection{General spectroscopic appearance}

The nightly average spectra for all systems are presented in Fig. 2. While the data for the three targets will be analysed individually in the subsequent sections, here we comment on the common spectroscopic properties. In all three objects, the WD is dominant in the blue part of the spectrum. EC 12477-1738 and EC 14329-1625 present broad and prominent Balmer absorption lines, while in EC 13349-3237 they are not as pronounced due to the system incorporating a much hotter WD (see the respective section).

In the red part, the late-type secondary star shows its presence in the form of a red continuum and molecular absorption bands. Again, while EC 12477-1738 and EC 14329-1625 at first glance present a very similar appearance, in EC 13349-3237 the less pronounced $\mathrm{TiO}$ band edge around $7050 \AA$ and the presence

2 version 07FEBpl1.1 on PC/Linux. of absorption features in the middle part of the spectrum suggest an earlier spectral type for the secondary star than in the other two systems.

We also find comparatively large differences for all spectra between one night and the other. Specifically, the April 3 data are about 0.4 mag brighter than the ones from April 5, and the continuum slopes appear redder on the second night. We have already commented in Sect. 2 on the non-photometric conditions during the observations, and these therefore can account for the brightness offset between the two nights. The difference in colour is probably due to atmospheric refraction, since the standard stars were observed at significantly different airmasses: LTT 4816 on April 3 at $M(z) \sim 1.7$ and LTT 7379 on April 5 close to the zenith. In contrast, the targets have been observed throughout the night, covering a wide range of airmasses, roughly from 2.0 to 1.0 for all three systems. We therefore conclude that the observed differences both in brightness and in colour are not intrinsic, but instead due to non-photometric conditions and atmospheric refraction. However, as we will see in the next section, EC 12477-1738 represents a special case.

\subsection{EC 12477-1738}

The photometric data present clear variability, but have insufficient coverage to pin down the orbital period, as is evident from the corresponding periodogram (Fig. 3a). The radial velocities of the $\mathrm{H} \alpha$ emission line, instead, yield a clear main peak at $f=2.76$ cyc d $^{-1}$, which corresponds to $P=0.362 \mathrm{~d}=8.70 \mathrm{~h}$ (Fig. 3b). The spectroscopic periodogram shows a number of alias periods with peak values larger than half the values of the strongest signal, which were also tested on the spectroscopic and photometric data. For all alias periods, at least one of the two phase-folded data sets showed strong discrepancies, especially regarding data from different nights, thus leaving the 


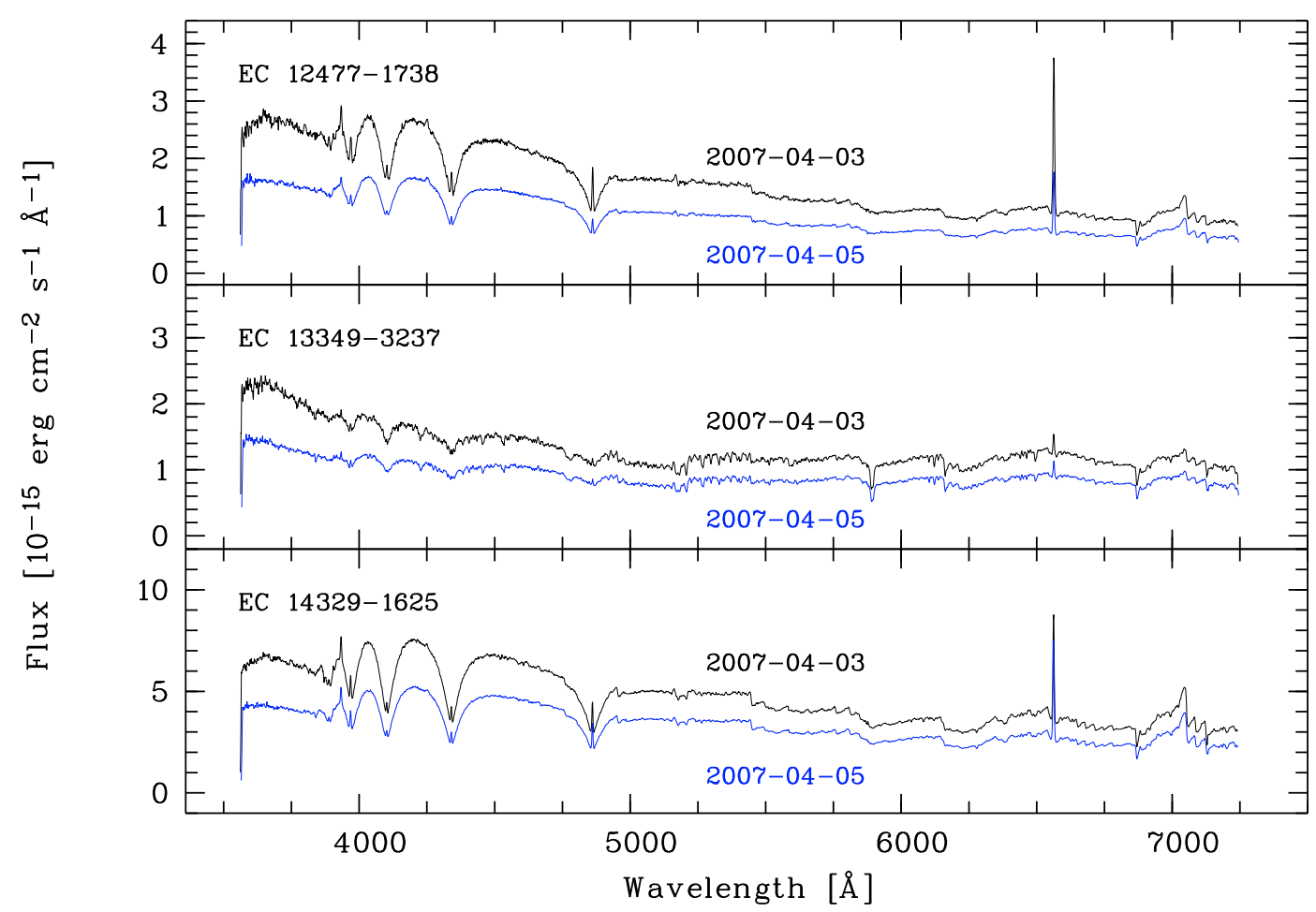

Fig. 2. Average spectra from April 3 and April 5, 2007 as labelled. Note the composite nature of all three systems, with the white dwarf dominating the blue part of the spectrum, and the late-type secondary star showing its presence in the red part. The narrow hydrogen emission lines also originate in the secondary star. The brightness and colour differences between the two nights are not intrinsic. See text for more detail.

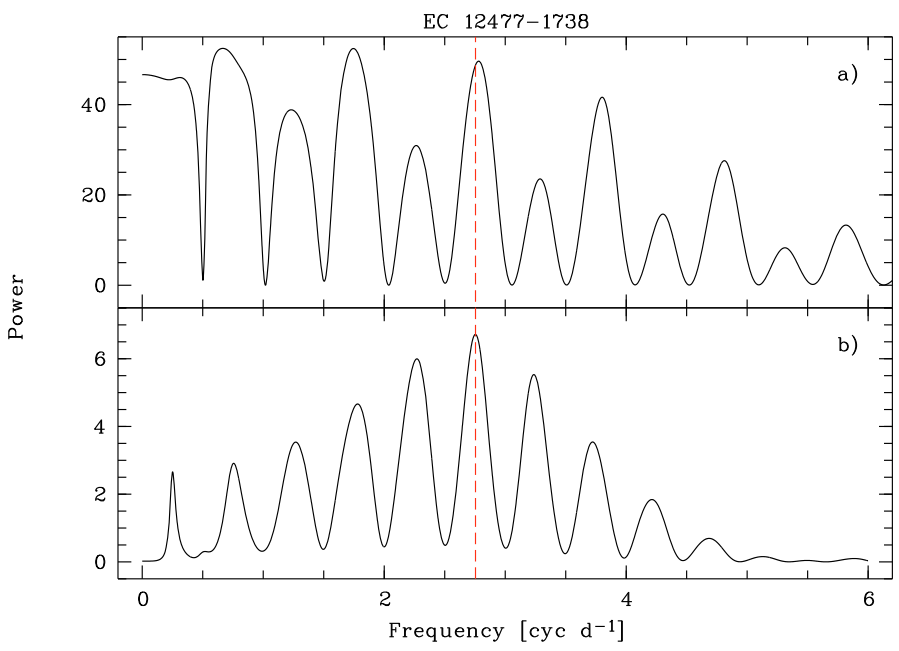

Fig. 3. Scargle periodograms for EC 12477-1738. a) Photometric data. b) Radial velocities of the $\mathrm{H} \alpha$ emission line. The hashed line marks the adopted period $P=0.362 \mathrm{~d}$.

aforementioned period corresponding to the strongest peak as the only viable choice.

Figures 4 and 5 give the phase-folded radial velocities and the light curve, respectively. The corresponding sine fit to the velocities $v(\varphi)$,

$v(\varphi)=\gamma+K_{2} \sin \varphi$

yields $\gamma=29(03) \mathrm{km} \mathrm{s}^{-1}, K_{2}=79(04) \mathrm{km} \mathrm{s}^{-1}$, and the ephemeris to

$T_{0}=$ HJD 2454 196.7045(25) + 0.362(08) E,

with $E$ giving the cycle number. This timing corresponds to the inferior conjunction of the emission source, i.e. most probably

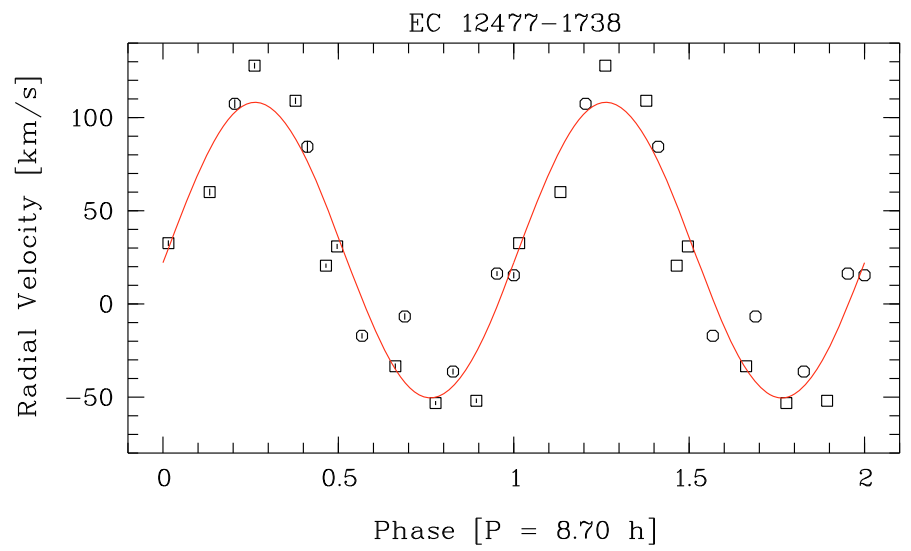

Fig. 4. Radial velocities of the $\mathrm{H} \alpha$ emission line for EC 12477-1738, phase-folded with respect to the ephemeris in Eq. (2). Circles mark data from 2007-04-03, squares those from 2007-04-05. The solid curve gives the best sine fit. The first cycle includes error bars corresponding to the Gaussian fit to the $\mathrm{H} \alpha$ emission line.

the secondary star. A recent radial velocity study by Maxted et al. (2007) determined $P=0.3664 \mathrm{~d}$, which agrees well with our result.

As explained in Sect. 3.1, the non-photometric conditions cause differences in brightness and continuum slopes between the two nights (Fig. 2, top). Folding the nightly average spectra with Bessell (1990) passbands, we find $V=16.0$ for April 3 and $V=16.5$ for April 5, while $B-V=0.2$ for both nights. Kilkenny et al. (1997) in their discovery paper give $V=16.20$. However, for EC 12477-1738 the emission lines with respect to the continuum also are significantly weaker. In Fig. 6 we see that, while the equivalent widths show some variation throughout both nights that does not appear to be correlated with the 


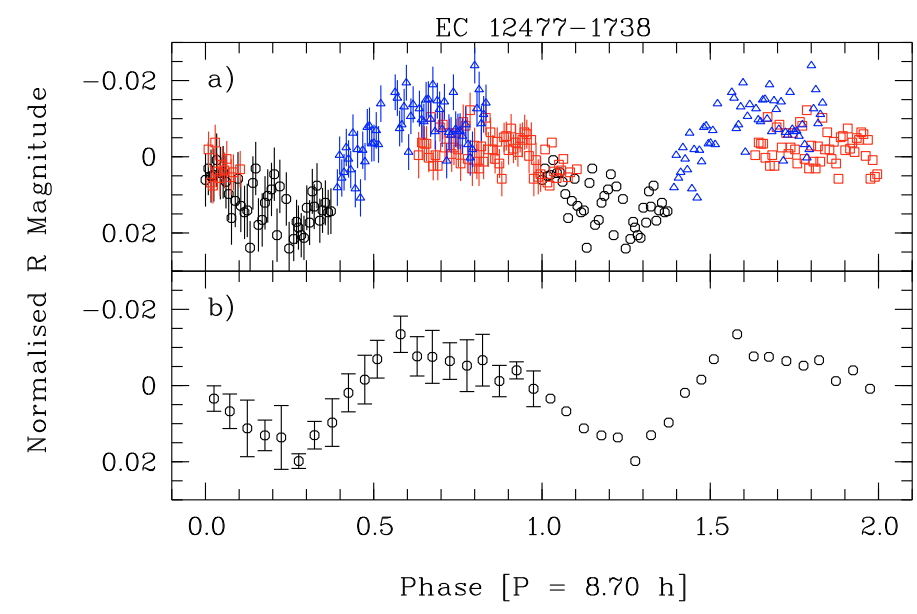

Fig. 5. Phase-folded light curves for EC 12477-1738, with phase zero arbitrarily set to the first data point. a) The individual data from April 08 (circles), 09 (squares), and 10 (triangles). Two cycles are shown, the second one without error bars. b) The data averaged into bins of 0.05 phases. The error bar gives the standard deviation with respect to the average value.

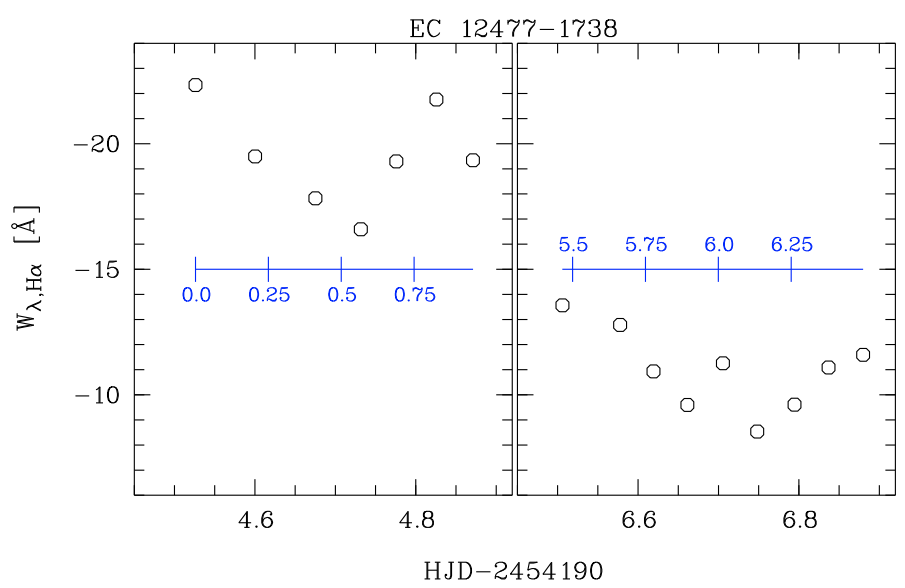

Fig. 6. $\mathrm{H} \alpha$ equivalent widths of the individual EC 12477-1738 spectra. The tickmarks give the phase with respect to $P=8.70 \mathrm{~h}$ (first data point $=$ phase 0.0 ).

orbital period, the average equivalent width drops to almost half its value from April 3 to April 5. The difference in the emission line strengths even probably makes up for the difference in the continuum slope between the two average spectra (as mentioned above, it is slightly bluer for April 3), thus yielding identical photometric colour indices for both nights, while for EC 133493237 and EC 14329-1625 the colour indices faithfully reflect the different continuum slopes.

The variable line strength suggests that EC 12477-1738 is another member of the group of pre-CVs with an active secondary star (e.g., O'Donoghue et al. 2003; Tappert et al. 2007a; van den Besselaar et al. 2007, for EC 13471-1258, LTT 560, and DE CVn, respectively). Further long-term photometric or spectroscopic monitoring will be needed for confirmation.

We have used the spectroscopic decomposition/fit procedure developed by Rebassa-Mansergas et al. (2007) to estimate the stellar parameters of the white dwarf and the companion star in EC 12477-1738. In brief, the observed pre-CV spectrum is first decomposed into a white dwarf and an M-dwarf component using a $\chi^{2}$-fit and a set of both white dwarf and M-dwarf template spectra from the SDSS. After subtracting the best-fit

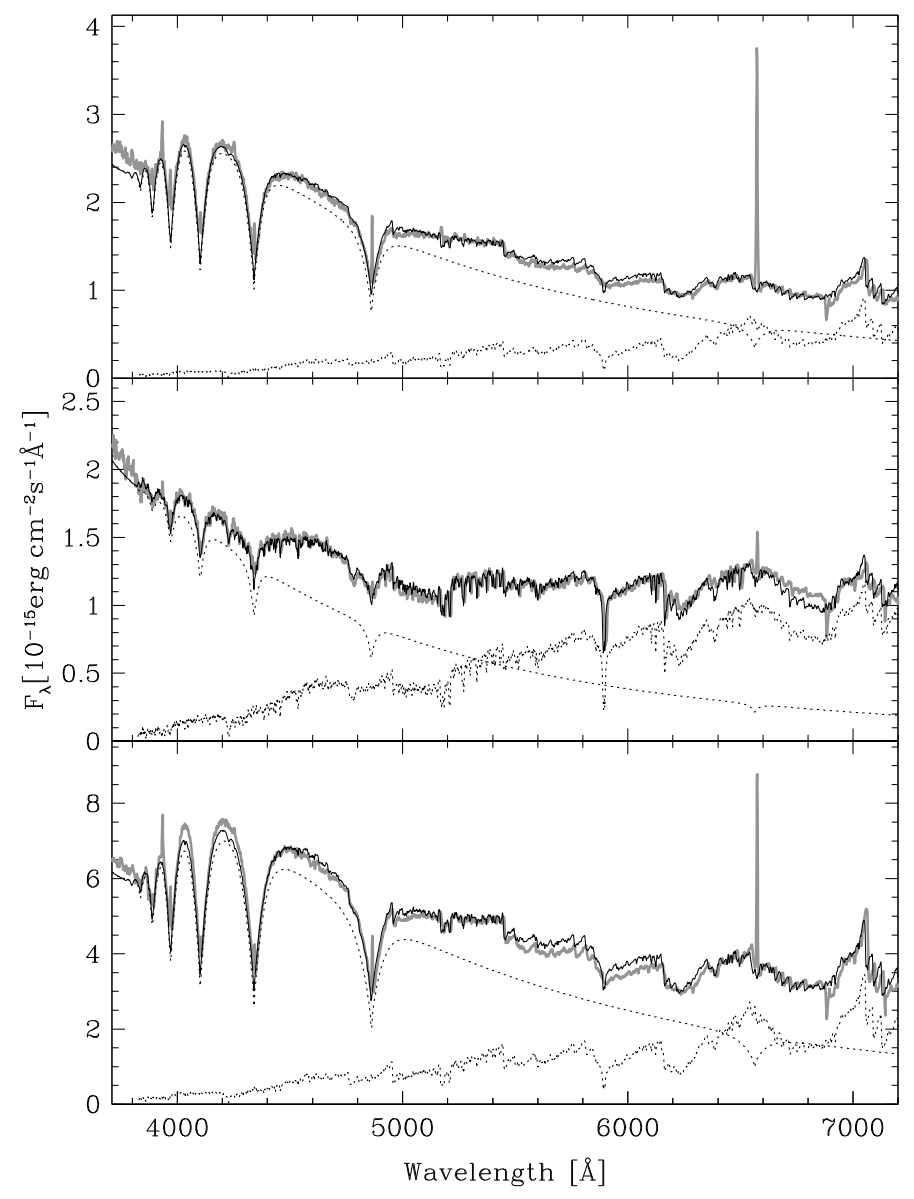

Fig. 7. Spectral fits to the spectra of EC 12477-1738 (top panel), EC 13349-3237 (middle panel) and EC 14329-1625 (bottom panel). The observed data are plotted as a thick gray line, the best-fit M-dwarf as a dotted line, and the best-fit white dwarf as hashed line. The sum of both components is overplotted on the data as a thin black line.

M-dwarf, the residual spectrum is then subjected to a fit with white dwarf model spectra computed using the code of Koester et al. (2005). The model fit is carried out on the normalised Balmer line profiles to avoid problems due to uncertainties in the response function of the spectrograph. Finally, the slope of the continuum is used to break the degeneracy between "cold" and "hot" model solutions that have approximatively equally strong Balmer absorption lines. For full details, we refer the reader to Rebassa-Mansergas et al. (2007). Free parameters in this decomposition/fit are the white dwarf temperature and surface gravity, which can be converted to a white dwarf mass using an updated version of the evolution sequences of Bergeron et al. (1995), the spectral type of the companion star, as well as distance estimates based on the flux scaling factors for both stellar components. The results from the decomposition of both spectra of EC 12477-1738 are reported in Table 3, and the composite fit to the April 3 spectrum is shown in Fig. 7 (top panel). Despite the different (and non-perfect) atmospheric conditions during both nights, both fits agree well within the errors, and suggest that EC 12477-1738 contains a white dwarf with a temperature of $T_{\mathrm{wd}}=17718 \pm 865 \mathrm{~K}$ and a mass close to the average mass of single white dwarfs (e.g. Liebert et al. 2005), $M_{\mathrm{wd}}=0.61 \pm 0.08 M_{\odot}$. The spectral type of the companion star is M3 \pm 0.5 . In principle, the two distance estimates determined from the flux scaling factor of each component should agree, but we find $d_{\mathrm{sec}}>d_{\mathrm{wd}}$. Rebassa-Mansergas et al. (2007) showed that 
Table 3. Stellar parameters for the white dwarfs and M-dwarfs in EC 12477-1738, EC 13349-3237 and EC 14329-1625 determined from a spectroscopic decomposition of the CTIO spectra.

\begin{tabular}{ccccccc}
\hline \hline & $T_{\mathrm{wd}}[\mathrm{K}]$ & $\log g$ & $M_{\mathrm{wd}}\left[M_{\odot}\right]$ & $d_{\mathrm{wd}}[\mathrm{pc}]$ & $\mathrm{Sp}(\mathrm{sec})$ & $d_{\mathrm{sec}}[\mathrm{pc}]$ \\
\hline EC 12477-1738 & $18330 \pm 1212$ & $8.08 \pm 0.26$ & $0.67 \pm 0.16$ & $111 \pm 19$ & $\mathrm{M} 3 \pm 0.5$ & $206 \pm 41$ \\
& $17106 \pm 1131$ & $7.88 \pm 0.26$ & $0.55 \pm 0.15$ & $151 \pm 24$ & $\mathrm{M} 3 \pm 0.5$ & $247 \pm 49$ \\
& $17718 \pm 865$ & $7.98 \pm 0.14$ & $0.61 \pm 0.08$ & $131 \pm 28$ & $M 3 \pm 0.5$ & $226 \pm 45$ \\
EC 13349-3237 & $37424 \pm 3880$ & $7.73 \pm 0.54$ & $0.54 \pm 0.28$ & $394 \pm 133$ & $\mathrm{M} 1 \pm 0.5$ & $321 \pm 63$ \\
& $32595 \pm 2052$ & $7.31 \pm 0.53$ & $0.38 \pm 0.19$ & $572 \pm 196$ & $\mathrm{M} 1 \pm 0.5$ & $373 \pm 73$ \\
& $35010 \pm 3415$ & $7.52 \pm 0.30$ & $0.46 \pm 0.11$ & $483 \pm 126$ & $M 1 \pm 0.5$ & $347 \pm 68$ \\
EC 14329-1625 & $13904 \pm 1409$ & $7.87 \pm 0.25$ & $0.54 \pm 0.15$ & $71 \pm 11$ & $\mathrm{M} 3 \pm 0.5$ & $120 \pm 24$ \\
& $15246 \pm 1299$ & $8.14 \pm 0.22$ & $0.70 \pm 0.14$ & $53 \pm 8$ & $\mathrm{M} 3 \pm 0.5$ & $106 \pm 21$ \\
& $14575 \pm 949$ & $8.01 \pm 0.19$ & $0.62 \pm 0.11$ & $62 \pm 13$ & $M 3 \pm 0.5$ & $113 \pm 10$ \\
\hline
\end{tabular}

Note: Three lines are given for each object, with the top (middle) line referring to the parameters determined from the spectra taken on April 3 (5) 2007. The bottom line, in italics, gives the average values and sample variance, except for $d_{\text {sec }}$, where the error is the average of the two individual errors.

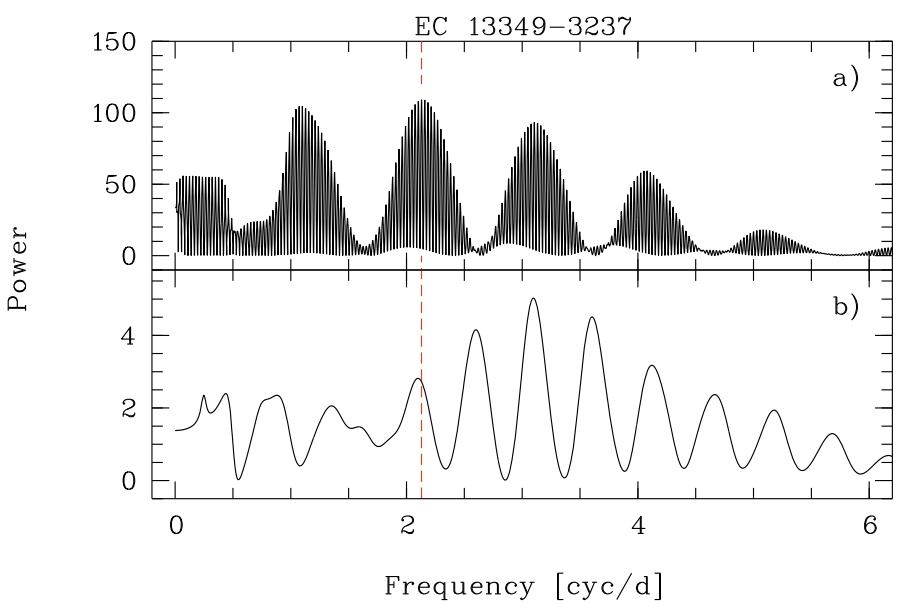

Fig. 8. Scargle periodograms for EC 13349-3237. a) Photometric data. b) Cross-correlation radial velocities of the absorption forest inbetween $\lambda 5163-5338$. The dashed line indicates the adopted period $P=0.4695 \mathrm{~d}$.

in about $\sim 1 / 3$ of the systems analysed in their study displayed the same problem. They discussed possible issues with the adopted spectral type-radius relation that may be related to stellar activity. Here we note that EC 12477-1738 exhibits strong emission line which vary on time scales of days, consistent with substantial chromospheric activity on the companion star.

\subsection{EC 13349-3237}

Four photometric data sets were taken in two different months, each time on two subsequent nights. The corresponding periodogram (Fig. 8, top) presents several hubs of fine-spaced alias periods. We can discard all hubs longwards of $f=2.5$, since the longest continuous data set of $6.28 \mathrm{~h}$ clearly does not represent 2/3 of an orbit (the triangles in Fig. 9). The hub shortwards of $f=1.5$ yields an ellipsoidal light curve at an orbital period $P \sim 22 \mathrm{~h}$. At a spectral type of M1 (see below) this would require an evolved secondary, and there is no spectroscopic evidence that would support such a scenario. The hub centred at $f=2.13$ therefore remains as the only possibility. We have folded the photometric data with all periods with peak values larger than half the value of the strongest peak, covering a frequency range $1.907-2.381 \mathrm{cyc} / \mathrm{d}$. Based on the criterion of how the data sets of different nights fit together in the phasefolded data, we find that only two periods, $P_{1}=0.4757 \mathrm{~d}$ and

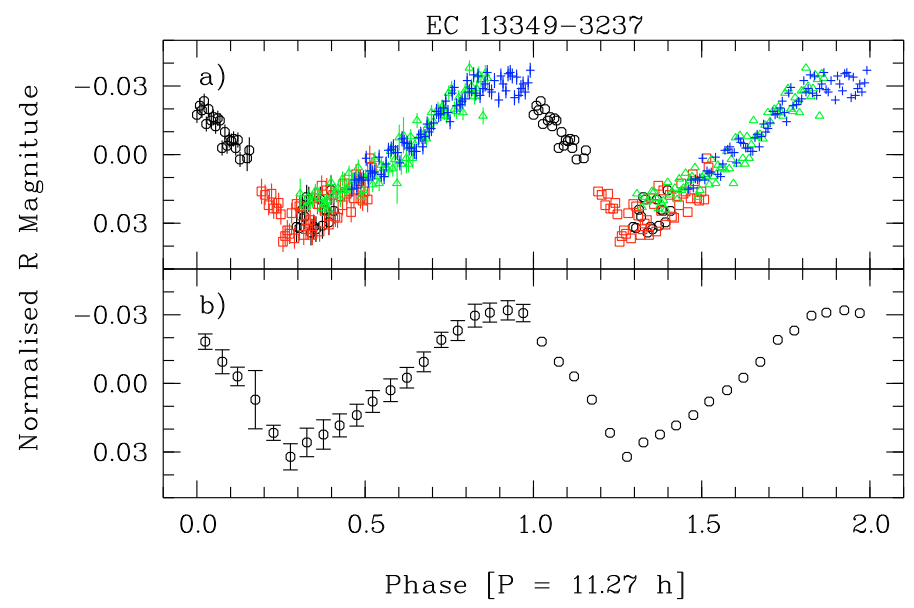

Fig. 9. Phase-folded light curves for EC 13349-3237. a) The individual data from May 15 (circles), 16 (squares), June 20 (triangles) and 11 (crosses). Two cycles are shown, the second one without error bars. b) The data averaged into bins of 0.05 phases. The error bar gives the standard deviation with respect to the average value.

$P_{2}=0.4695 \mathrm{~d}$ yield an acceptable light curve. Since $P_{2}$ is the slightly stronger one of the two, we adopt as photometric period $P_{\mathrm{ph}}=0.4695(01) \mathrm{d}$. As a word of caution we remark that our criterion here assumes that each data set represents a part of a stable, identical light curve. However, the potential presence of star spots or activity on the secondary star could induce a certain variability of the light curve. This applies to all three targets of this study, but bears special importance for EC 13 349-3237, as here we are dealing with 4 incomplete parts of a light curve within two data sets that are separated by one month.

Somewhat surprisingly, the spectroscopic data do not present a similarly clear variation, and in fact do not appear to reflect the photometric variation at all. Measuring radial velocities by fitting single Gaussians to the $\mathrm{H} \alpha$ emission line or to a number of absorption lines ( $\mathrm{Na} \mathrm{I} \lambda 5893, \mathrm{Ca} \mathrm{I} \lambda 6103$ and $\lambda 6122)$ results in very noisy curves without any clear periodic signal. In a second attempt we measured radial velocities by cross-correlation in the spectral region 5160-5340 A, which contains a forest of absorption lines from the secondary star due to $\mathrm{Mg}, \mathrm{Cr}$ and $\mathrm{Fe}$. We used a synthetic template spectrum to avoid introducing additional noise into the results. The template was calculated using the UCLSYN code (Smalley et al. 2001) and adopting $T_{\text {eff }}=3500 \mathrm{~K}$ and $\log g=4.5$. This yielded radial velocities which were less noisy but still did not demonstrate the expected variations in that 


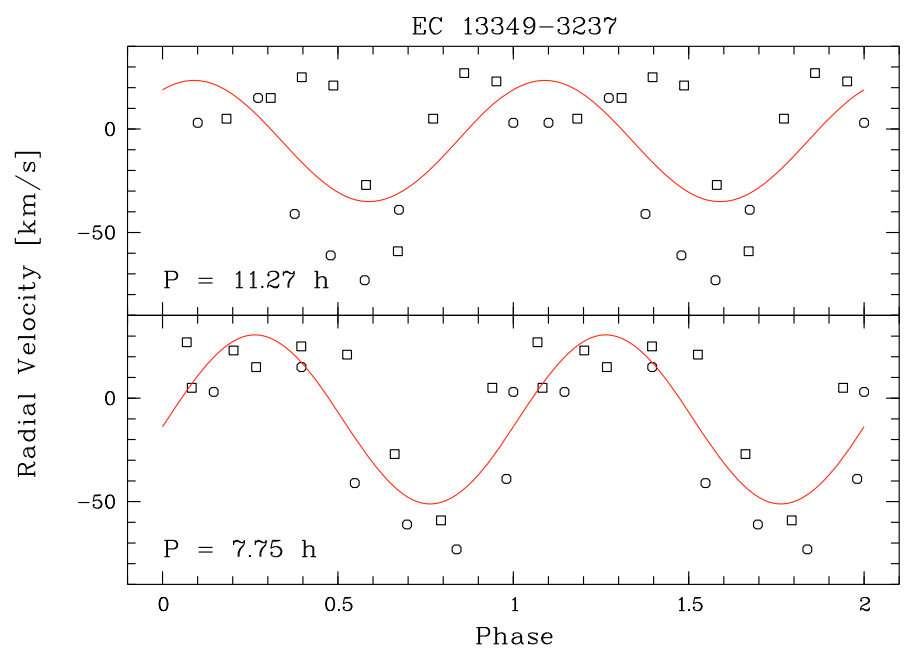

Fig. 10. EC 13349-3237 cross-correlation radial velocities folded on the photometric period (top) and on the "spectroscopic" period (bottom). Symbols are the same as in Fig. 11. The lines shows the best sinusoidal fit with parameters given in Table 4.

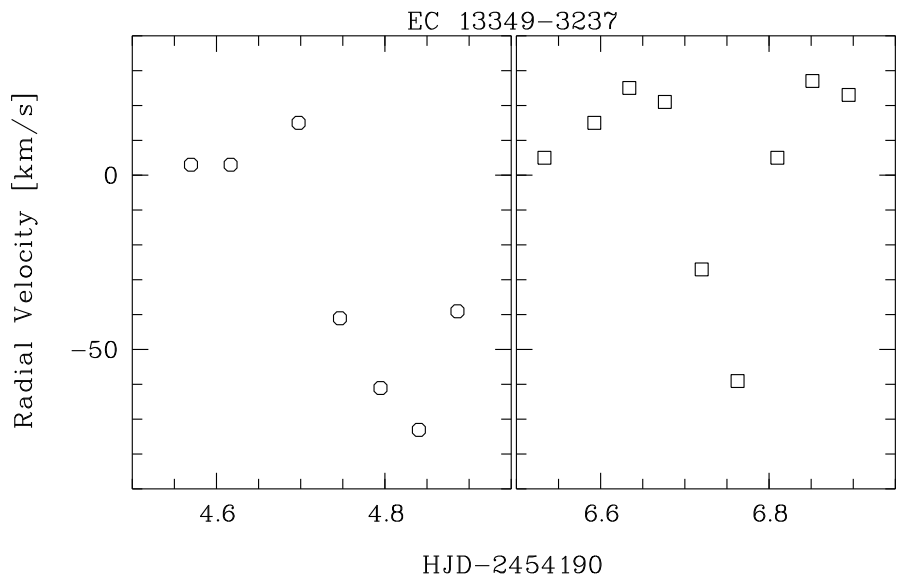

Fig. 11. EC 13349-3237 radial velocities, as determined by a crosscorrelation of the absorption forest in-between $\lambda 5163-5338 \AA$ with a synthetic template.

they do not appear to follow the photometric period, but instead prefer $P=0.323 \mathrm{~d}$ (Fig. 8, bottom).

We have folded the radial velocity data on both the photometric period and the one extracted from the spectroscopic periodogram. As expected, since the photometric period is barely, if at all, present in the spectroscopic data, that period yields a very poor fit (Fig. 10, top). The "spectroscopic" period at first glance provides an acceptable fit to the data (Fig. 10, bottom). However, closer inspection reveals that there are systematic differences between the data from the two nights, as, with one exception, the velocities from the first night all lie below the fit. In Fig. 11 we have plotted the radial velocities in sequence versus time, which makes it even more obvious that the velocities do not follow a well-defined sinusoidal variation. We therefore doubt the physical relevance of this signal. Again we point out that the longest photometric data set excludes the "spectroscopic" period for the light curve.

Without more and better data, we are not able to clarify this puzzling behaviour. Perhaps it is due to a combination of the low spectral resolution and a low inclination (for the photometric variation the low inclination could be compensated for by a particularly strong reflection effect due to a hot white

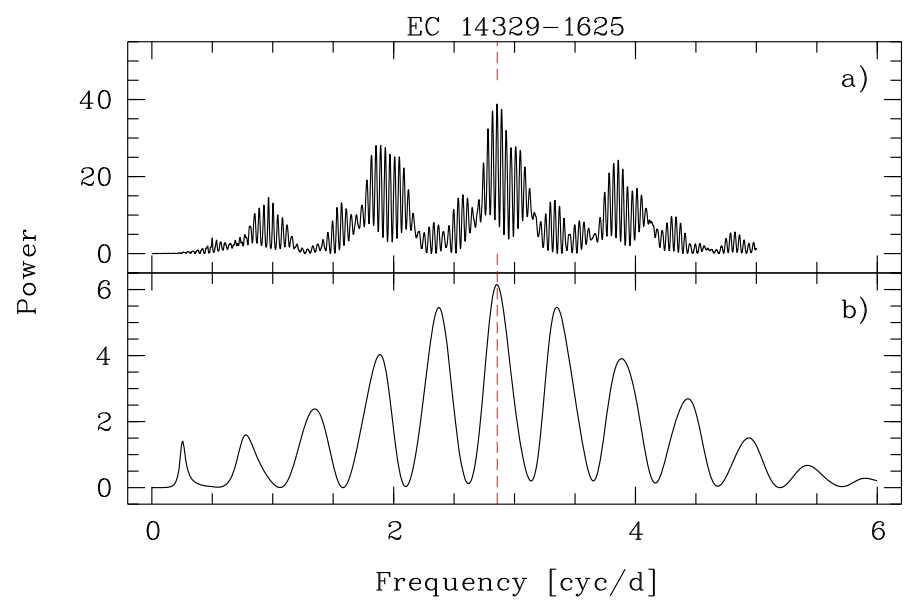

Fig. 12. Scargle periodograms for EC 14329-1625. a) 2005 photometric data. b) $\mathrm{H} \alpha$ radial velocities. The hashed line marks the adopted period $P=0.3500 \mathrm{~d}$.

dwarf). Further investigation of this system clearly requires time-resolved high-resolution spectroscopy.

Folding the nightly average spectra with Bessell filters we obtain $V=16.26$ and $B-V=0.36$ for April 3, and $V=16.61$, $B-V=0.42$ for April 5. The difference in magnitude is very similar to that found for EC 12477-1738, and we attribute this and the difference in the continuum slope to the non-photometric conditions during the observations. Previously reported values for EC 13 349-3237 are $V=16.34, B-V=0.36$ (Kilkenny et al. 1997).

Using the spectroscopic decomposition/fit technique introduced in Sect. 3.2, we determine the white dwarf temperature and mass of EC 13349-3237, $T_{\mathrm{wd}}=35010 \pm 3415 \mathrm{~K}$, and $M_{\mathrm{wd}}=0.46 \pm 0.11$ (Table 3 and Fig. 7, middle panel). Taken at face value, the white dwarf mass is lower than the average mass of single white dwarfs, suggestive of a He-core as a result of the common envelope evolution. Only a handful of bona-fide $\mathrm{He}$-core white dwarfs in pre-CVs are known, and a more detailed study appears warranted to confirm this hypothesis for EC 13349-3237. The spectral type of the companion, $\mathrm{Sp}(\mathrm{sec})=\mathrm{M} 1 \pm 0.5$. Hence, EC $13349-3237$ is a new addition to the still very small number of pre-CVs with early-type companion stars that will start mass transfer above the period gap (Schreiber \& Gänsicke 2003). The distances determined for the two components are in good agreement.

\subsection{EC 14329-1625}

This is the brightest of the three pre-CVs of our study, and this made it possible to also use the photometric data from the 2005 runs which suffered from bad weather conditions. Unfortunately, the time span between both sets (from 2003 and 2005) is too long for a combined period search. The periodogram of the 2005 data yields as the most probable pe$\operatorname{riod} P_{1}=0.3500(08) \mathrm{d}$, that agrees well with the result $P_{\mathrm{sp}}=$ $0.3498(35) \mathrm{d}$ from the radial velocities of the $\mathrm{H} \alpha$ emission line from the 2007 time-resolved spectroscopy (Fig. 12). The phase-folded photometric and spectroscopic data are given in Figs. 13 and 14, respectively. The second highest peak for the photometric data, $P_{2}=0.3457(08) \mathrm{d}$, yields a visually equally good light curve, and further observations will be necessary to definitely break this alias degeneracy. However, since $P_{1}$ agrees slightly better with the spectroscopic period, we adopt 


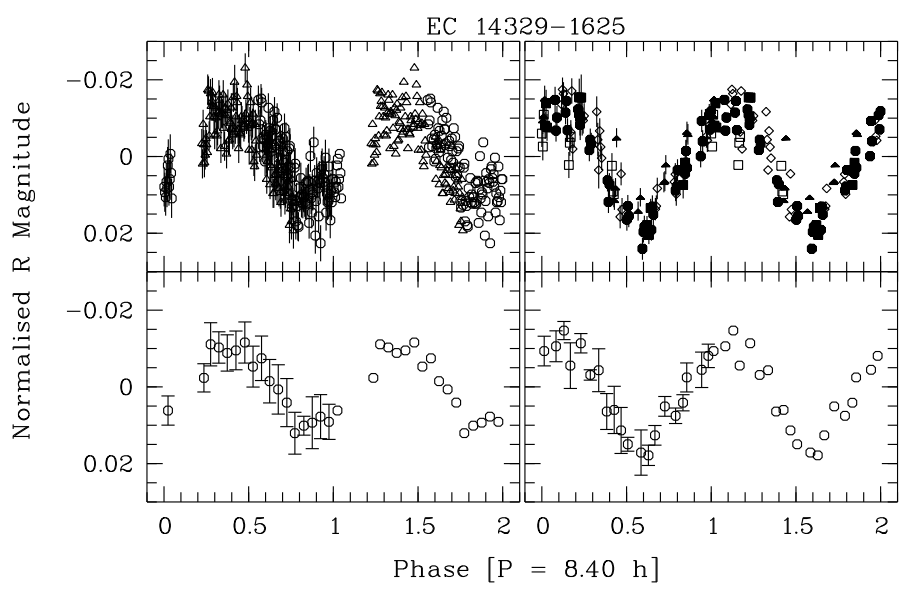

Fig. 13. EC 14329-1625 phase-folded light curves from 2003 (left) and 2005 (right). Different symbols mark data from different nights. The bottom plots show the respective data averaged into 0.05 phase bins.

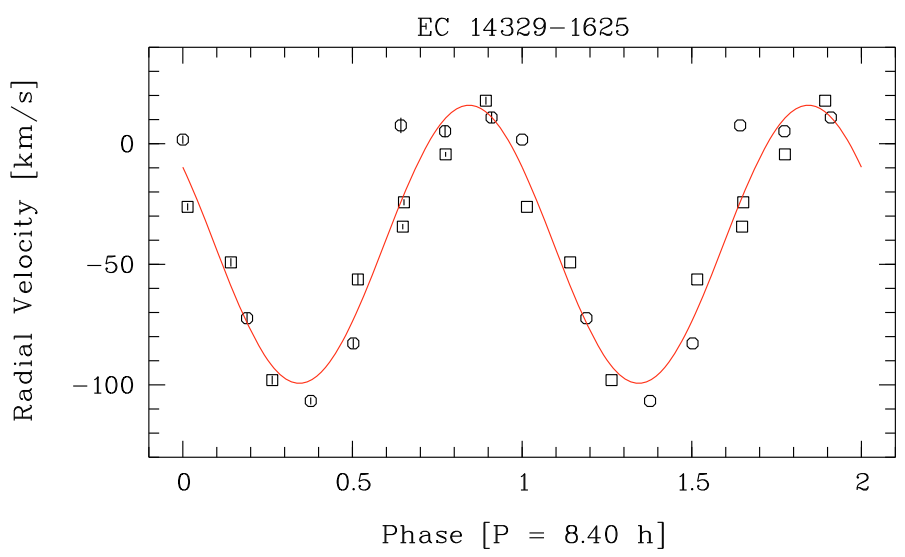

Fig. 14. Phase-folded radial velocities of the $\mathrm{H} \alpha$ emission line for EC 14329-1625. Circles mark data from 2007-04-03, squares those from 2007-04-05. The solid curve gives the best sine fit. The first cycle includes error bars corresponding to the Gaussian fit to the $\mathrm{H} \alpha$ emission line.

$P_{\text {orb }}=0.3500(08) \mathrm{d}=8.40(02) \mathrm{h}$ as the orbital period of EC 14329-1625. A sine fit to the radial velocity data yield the parameters listed in Table 4, and the corresponding ephemeris is

$T_{0}=$ HJD $2454196.5437(24)+0.3500(08) E$,

with respect to the inferior conjunction of the emission source.

As for the other two targets, the average spectra show a flux difference between the two nights (Fig. 2, bottom). Folding the data with Bessell filter curves yields similar differences as for the other systems, with values $V=14.79, B-V=0.33$ for April 3, and $V=15.13, B-V=0.38$ for April 5. Kilkenny et al. (1997) report $V=14.89, B-V=0.25$, and again the April 3 data agree slightly better with their measurements.

For EC 14329-1625, the spectroscopic decomposition (see Sect. 3.2) results in $T_{\mathrm{wd}}=14600 \pm 1300 \mathrm{~K}$ and $M_{\mathrm{wd}}=0.62 \pm 0.14$ (Table 3 and Fig. 7, middle panel), and similar to EC $12477-$ 1738, EC 14329-1625 has a mass close to the average mass of single white dwarfs. Another similarity to EC $12477-1738$ is that we find $d_{\mathrm{sec}}>d_{\mathrm{wd}}$, which may suggest that the companion star has a radius slightly too large for its spectral type. As already mentioned in Sect. 3.2, this might be related to stellar activity on the companion star (Rebassa-Mansergas et al. 2007). In fact, both EC 12 477-1738 and EC 14 329-1625 exhibit very strong Balmer emission lines. Given their long orbital periods
Table 4. Radial-velocity parameters: the adopted orbital period $P$, the semi-amplitude $K_{2}$, the constant term $\gamma$, and the standard deviation of the fit $\sigma$.

\begin{tabular}{lllll}
\hline \hline Object & $P$ & $K_{2}$ & $\gamma$ & $\sigma$ \\
& {$[\mathrm{d}]$} & {$\left[\mathrm{km} \mathrm{s}^{-1}\right]$} & {$\left[\mathrm{km} \mathrm{s}^{-1}\right]$} & {$\left[\mathrm{km} \mathrm{s}^{-1}\right]$} \\
\hline EC 12477-1738 & $0.362(08)$ & $79(04)$ & $29(03)$ & 19 \\
EC 13349-3237 & $0.4695(01)$ & {$[29(06)$} & $-6(04)$ & $27]$ \\
& $0.4757(01)^{1}$ & & & \\
& {$[0.323(08)$} & $41(04)$ & $-10(03)$ & $20]^{2}$ \\
EC 14329-1625 & $0.3500(08)$ & $58(03)$ & $-42(02)$ & 12 \\
& $0.3457(08)^{1}$ & & & \\
\hline
\end{tabular}

1) best alias period, yielding an equally acceptable light curve; 2) the "spectroscopic" period and the radial velocity parameters are formally included, but should be taken with great caution, due to the radialvelocity variation being not well understood

and modest white dwarf temperatures, the strength of the Balmer lines is indicative of chromospheric activity, rather than irradiation/heating of the companion star.

\section{Conclusions}

We have determined the system parameters for the three preCVs EC 12477-1738, EC 13349-3237, and EC 14329-1625. The results of the spectroscopic decomposition are given in Table 3, the parameters gained from the radial velocities are summarised in Table 4. When comparing our results with previously published data we find that Koester et al. (2001) determine somewhat higher temperatures for the WDs in EC 12477$1738(20922 \pm 317 \mathrm{~K}$ vs. $17718 \pm 865 \mathrm{~K})$ and in EC 133493237 (48 116 $\pm 1353 \mathrm{~K}$ vs. $35010 \pm 3000 \mathrm{~K})$. These differences are explained by the fact that Koester et al. (2001) did not correct for the contribution of the companion star before fitting the white dwarf spectrum. Consequently, the equivalent widths of the white dwarf photospheric Balmer lines are underestimated, which pulls the spectroscopic fit to higher temperatures (a higher degree of ionisation).

The secondary spectral types for all three pre-CVs agree well with those obtained by Tappert et al. (2007b) from absorption line strengths in $K$-band spectra: M3V (this study: M3 $\pm 0.5 \mathrm{~V}$ ) for EC 12477-1738 and EC 14329-1625, and K5V-M2V (M1 \pm $0.5 \mathrm{~V})$ for EC 13349-3237.

Two of our systems, EC 12477-1738 and EC 14329-1625, turn out to have very similar parameters: their orbital period is close to $8.5 \mathrm{~h}$, and their stellar components are a medium-hot WD and an M3V secondary. The latter is an unusual combination for the pre-SDSS sample of pre-CVs, which was strongly biased to hotter WDs (Schreiber \& Gänsicke 2003), but it is now found quite frequently in pre-CVs discovered in the SDSS (Rebassa-Mansergas et al. 2007). EC 13349-3237, on the other hand, while submitting to the usual observational bias in being a young pre-CV with a hot $\mathrm{WD}$, also incorporates a comparatively early-type, M1V, secondary star. Pre-CVs with an earlytype secondary star still represent a minority in the currently known sample. As Schreiber \& Gänsicke (2003) point out, this is due to the fact that the secondary star in these systems contributes too much light for the object to appear as a candidate WD or QSO in the colour-colour diagram and thus will not trigger spectroscopic follow-up observations. It is probable that, if EC 13349-3237 contained a cooler WD, it would still remain undiscovered (see also Schreiber et al. 2007). 
Based on the spectral type of the secondary stars, we can expect that EC 12477-1738 and EC 14329-1625 will turn into CVs with an orbital period 3-5 h, while EC 13349-3237 will enter its CV phase at $P \sim 4-6$ h (e.g., Beuermann et al. 1998). In this context it is worth noticing that the period regime at 3$4 \mathrm{~h}$ is dominated by the high-mass-transfer SW Sex stars, whose physics and possible magnetic nature are still under debate (Rodríguez-Gil et al. 2007, and references herein). A detailed examination of pre-CVs with secondary spectral types $\sim \mathrm{M} 3 \mathrm{~V}$, such as EC 12477-1738 and EC 14329-1625, might therefore provide valuable insight into this important subgroup of CVs.

Acknowledgements. We thank the anonymous referee for comments that helped to improve the paper. C.T. and R.E.M. acknowledge financial support by FONDECYT grant 1051078. R.E.M. also acknowledges financial support by the Chilean Center for Astrophysics FONDAP 15010003 and from the BASAL Centro de Astrofísica y Tecnologias Afines (CATA) PFB-06/2007. We thank Detlev Koester for providing us his white dwarf model spectra. This work has made intensive use of the SIMBAD database, operated at CDS, Strasbourg, France, and of NASA's Astrophysics Data System Bibliographic Services. The Digitized Sky Surveys were produced at the Space Telescope Science Institute under US Government grant NAG W-2166, based on photographic data obtained using the Oschin Schmidt Telescope on Palomar Mountain and the UK Schmidt Telescope. IRAF is distributed by the National Optical Astronomy Observatories.

\section{References}

Bergeron, P., Wesemael, F., \& Beauchamp, A. 1995, PASP, 107, 1047 Bessell, M. S. 1990, PASP, 102, 1181

Beuermann, K., Baraffe, I., Kolb, U., \& Weichhold, M. 1998, A\&A, 339, 518

Broeg, C., Fernández, M., \& Neuhäuser, R. 2005, Astron. Nachr., 326, 134

Gänsicke, B. T. 2005, The Astrophysics of Cataclysmic Variables and Related Objects, ed. J.-M. Hameury \& J.-P. Lasota, ASP Conf. Ser., 330

Gänsicke, B. T., Szkody, P., de Martino, D., et al. 2003, ApJ, 594, 443

Harrison, T. E., Osborne, H. L., \& Howell, S. B. 2004, AJ, 127, 3493

Harrison, T. E., Osborne, H. L., \& Howell, S. B. 2005, AJ, 129, 2400

Horne, K. 1986, PASP, 98, 609

Kilkenny, D., O’Donoghue, D., Koen, C., Stobie, R. S., \& Chen, A. 1997, MNRAS, 287, 867

Koester, D., Napiwotzki, R., Christlieb, N., et al. 2001, A\&A, 378, 556
Koester, D., Napiwotzki, R., Voss, B., Homeier, D., \& Reimers, D. 2005, A\&A, 439,317

Larsson, S. 1996, A\&AS, 117, 197

Liebert, J., Bergeron, P., \& Holberg, J. B. 2005, ApJS, 156, 47

Maxted, P. F. L., Napiwotzki, R., Marsh, T. R., et al. 2007, in 15th European Workshop on White Dwarfs, ed. R. Napiwotzki \& M. R. Burleigh, ASP Conf. Ser., 372, 471

Napiwotzki, R., \& Burleigh, M. R., 2007, 15th European Workshop on White Dwarfs, ASP Conf. Ser., 372

O’Donoghue, D., Koen, C., Kilkenny, D., et al. 2003, MNRAS, 345, 506

Patterson, J. 1998, PASP, 110, 1132

Raymond, S. N., Szkody, P., Hawley, S. L., et al. 2003, AJ, 125, 2621

Rebassa-Mansergas, A., Gänsicke, B. T., Rodríguez-Gil, P., Schreiber, M. R., \& Koester, D. 2007, MNRAS, 382, 1377

Ritter, H., \& Kolb, U. 2003, A\&A, 404, 301

Rodríguez-Gil, P., Gänsicke, B. T., Hagen, H.-J., et al. 2007, MNRAS, 377, 1747

Scargle, J. D. 1982, ApJ, 263, 835

Schenker, K., \& King, A. R. 2002, in The Physics of Cataclysmic Variables and Related Objects, ed. B. T. Gänsicke, K. Beuermann, \& K. Reinsch, ASP Conf. Ser., 261, 242

Schenker, K., King, A. R., Kolb, U., Wynn, G. A., \& Zhang, Z. 2002, MNRAS, 337,1105

Schreiber, M., Nebot Gomez-Moran, A., \& Schwope, A. 2007, in 15th European Workshop on White Dwarfs, ed. R. Napiwotzki \& M. R. Burleigh, ASP Conf. Ser., 372, 459

Schreiber, M. R., \& Gänsicke, B. T. 2003, A\&A, 406, 305

Schreiber, M. R., Gänsicke, B. T., Southworth, J., Schwope, A. D., \& Koester, D. 2008, A\&A, 484, 441

Schwarzenberg-Czerny, A. 1989, MNRAS, 241, 153

Schwarzenberg-Czerny, A. 1996, ApJ, 460, L107

Silvestri, N. M., Hawley, S. L., West, A. A., et al. 2006, AJ, 131, 1674

Silvestri, N. M., Lemagie, M. P., Hawley, S. L., et al. 2007, AJ, 134, 741

Smalley, B., Smith, K., \& Dworetsky, M. 2001, UCLSYN Userguide

Stehle, R., Kolb, U., \& Ritter, H. 1997, A\&A, 320, 136

Stetson, P. B. 1992, in Astronomical Data Analysis Software and Systems I, ed. D. M. Worrall, C. Biemesderfer, \& J. Barnes, ASP Conf. Ser., 25, 297

Tappert, C., Gänsicke, B. T., \& Mennickent, R. E. 2004, in Compact Binaries in the Galaxy and Beyond, ed. G. Tovmassian \& E. Sion, Rev. Mex. Astron. Astrofis. Conf. Ser., 20, 245

Tappert, C., Gänsicke, B. T., Schmidtobreick, L., et al. 2007a, A\&A, 474, 205

Tappert, C., Gänsicke, B. T., Schmidtobreick, L., Mennickent, R. E., \& Navarrete, F. P. 2007b, A\&A, 475, 575

Tappert, C., Toledo, I., Gänsicke, B. T., \& Mennickent, R. E. 2006, in XI IAU Regional Latin American Meeting of Astronomy, ed. L. Infante \& M. Rubio, Rev. Mex. Astrono. Astrofis. Conf. Ser., 26, 177

van den Besselaar, E. J. M., Greimel, R., Morales-Rueda, L., et al. 2007, A\&A, 466, 1031 